Research Article

\title{
Some Existence Results of Positive Solutions for $\varphi$-Laplacian Systems
}

\author{
Xianghui Xu and Yong-Hoon Lee \\ Department of Mathematics, Pusan National University, Busan 609-735, Republic of Korea \\ Correspondence should be addressed to Yong-Hoon Lee; yhlee@pusan.ac.kr \\ Received 29 January 2014; Accepted 7 April 2014; Published 4 May 2014 \\ Academic Editor: Julio D. Rossi
}

Copyright (c) 2014 X. Xu and Y.-H. Lee. This is an open access article distributed under the Creative Commons Attribution License, which permits unrestricted use, distribution, and reproduction in any medium, provided the original work is properly cited.

We study the existence of positive solutions for the homogeneous Dirichlet boundary value problem of $\varphi$-Laplacian systems with a singular weight which may not be in $L^{1}$.

\section{Introduction}

In this paper, we study nonlinear differential systems of the form

$$
\begin{gathered}
-\Phi\left(\mathbf{u}^{\prime}\right)^{\prime}=\mathbf{h}(t) \cdot \mathbf{f}(\mathbf{u}), \quad t \in(0,1) \\
\mathbf{u}(0)=0=\mathbf{u}(1)
\end{gathered}
$$

where $\Phi\left(\mathbf{u}^{\prime}\right)=\left(\varphi\left(u_{1}^{\prime}\right), \ldots, \varphi\left(u_{N}^{\prime}\right)\right)$ with $\varphi: \mathbb{R} \rightarrow \mathbb{R}$ an odd increasing homeomorphism, $\mathbf{h}(t)=\left(h_{1}(t), \ldots, h_{N}(t)\right)$ with $h_{i}:(0,1) \rightarrow \mathbb{R}_{+}, h_{i} \not \equiv \quad 0$ on any subinterval in $(0,1)$, and $\mathbf{f}(\mathbf{u})=\left(f^{1}(\mathbf{u}), \ldots, f^{N}(\mathbf{u})\right)$ with $f^{i}: \mathbb{R}_{+}^{N} \rightarrow \mathbb{R}_{+} ;$ here we denote $\mathbb{R}_{+}=[0,+\infty), \mathbb{R}_{+}^{N}=\underbrace{\mathbb{R}_{+} \times \cdots \times \mathbb{R}_{+}}_{N}$, and $\mathbf{x} \cdot \mathbf{y}=\left(x_{1} y_{1}, x_{2} y_{2}, \ldots, x_{N} y_{N}\right)$ the Hadamard product of $\mathbf{x}$ and $\mathbf{y}$ in $\mathbb{R}^{N}$. Thus problem $(P)$ can be rewritten as

$$
\begin{aligned}
&-\varphi\left(u_{1}^{\prime}\right)^{\prime}=h_{1}(t) f^{1}(\mathbf{u}), \\
& \vdots \\
&-\varphi\left(u_{N}^{\prime}\right)^{\prime}=h_{N}(t) f^{N}(\mathbf{u}), \quad t \in(0,1), \\
& u_{i}(0)=0=u_{i}(1), \quad i=1, \ldots, N .
\end{aligned}
$$

We first give assumptions on $\varphi$ and $\mathbf{h}$.

(A) There exist an increasing homeomorphism $\psi$ of $(0, \infty)$ onto $(0, \infty)$ and a function $\gamma$ of $(0, \infty)$ into $(0, \infty)$ such that

$$
\psi(\sigma) \leq \frac{\varphi(\sigma x)}{\varphi(x)} \leq \gamma(\sigma), \quad \forall \sigma>0, x \in \mathbb{R} .
$$

$(\mathrm{H}) h_{i}:(0,1) \rightarrow \mathbb{R}_{+}$is locally integrable satisfying

$$
\begin{aligned}
& \int_{0}^{1 / 2} \psi^{-1}\left(\int_{s}^{1 / 2} h_{i}(\tau) d \tau\right) d s+\int_{1 / 2}^{1} \psi^{-1}\left(\int_{1 / 2}^{s} h_{i}(\tau) d \tau\right) d s \\
& <\infty
\end{aligned}
$$

$$
\text { for } i=1, \ldots, N \text {. }
$$

For convenience, we introduce a new class of weight functions. For a bijection $\iota: \mathbb{R} \rightarrow \mathbb{R}$, define $\mathscr{H}_{\iota}$ as a subset of $L_{\text {loc }}^{1}\left((0,1), \mathbb{R}_{+}\right)$given by

$$
\begin{aligned}
\mathscr{H}_{\iota}=\left\{g \mid \int_{0}^{1 / 2} \iota^{-1}\left(\int_{s}^{1 / 2} g(\tau) d \tau\right) d s\right. \\
\left.+\int_{1 / 2}^{1} \iota^{-1}\left(\int_{1 / 2}^{s} g(\tau) d \tau\right) d s<\infty\right\} .
\end{aligned}
$$

By the notation, condition $(\mathrm{H})$ means $h_{i} \in \mathscr{H}_{\psi}$. 
The case of $p$-Laplace operator, namely, $\varphi(x)=\varphi_{p}(x):=$ $|x|^{p-2} x, x \in \mathbb{R}, p>1$, satisfies condition (A) with $\psi \equiv \varphi_{p} \equiv \gamma$. We give one more example of $\varphi$ and $\mathbf{h}$ satisfying conditions (A) and (H).

Example 1. Define $\varphi: \mathbb{R} \rightarrow \mathbb{R}$ as an odd function with

$$
\varphi(x)=x^{2}+x, \quad x \geq 0 .
$$

Then $\varphi$ is obviously an increasing homeomorphism. Define functions $\psi$ and $\gamma$ given as

$$
\begin{aligned}
& \psi(\sigma)= \begin{cases}\sigma^{2}, & \text { if } 0<\sigma \leq 1, \\
\sigma, & \text { if } \sigma>1,\end{cases} \\
& \gamma(\sigma)= \begin{cases}1, & \text { if } 0<\sigma \leq 1, \\
\sigma^{2}, & \text { if } \sigma>1 .\end{cases}
\end{aligned}
$$

Then $\psi, \gamma:(0, \infty) \rightarrow(0, \infty)$ and $\psi$ is an increasing homeomorphism. This implies that $\varphi$ satisfies condition (A). Moreover, for $h(t)=t^{-3 / 2}$, we can easily calculate to see $h \in \mathscr{H}_{\psi}$.

We note that $h$ given in the example above is not integrable near a boundary $t=0$; that is, $h \notin L^{1}(0,1)$, and, in this paper, we focus on studying generalized Laplacian systems of condition $(\mathrm{A})$ with singular weights which may not be in $L^{1}(0,1)$. We now give assumptions on $\mathbf{f}$.

(F) $f^{i}: \mathbb{R}_{+}^{N} \rightarrow \mathbb{R}_{+}$is continuous, $i=1, \ldots, N$.

Problems of $p$-Laplacian or more generalized ones like problem $(P)$ appear in various applications which describe reaction-diffusion systems, nonlinear elasticity, glaciology, population biology, combustion theory, and non-Newtonian fluids (see [1-4]). Recently there is a vast literature related to existence, multiplicity, or nonexistence of positive solutions of problem $(P)$ for either $p$-Laplacian or more generalized Laplacian problems (see [5-11] and the references therein). Specially, for generalized Laplacian problems, one may refer to works of Agarwal et al. (see [12-14]). Let us denote

$$
\mathbf{f}_{0}:=\sum_{i=1}^{N} f_{0}^{i}, \quad \mathbf{f}_{\infty}:=\sum_{i=1}^{N} f_{\infty}^{i}
$$

where

$$
f_{0}^{i}:=\lim _{\|\mathbf{u}\| \rightarrow 0} \frac{f^{i}(\mathbf{u})}{\varphi(\|\mathbf{u}\|)}, \quad f_{\infty}^{i}:=\lim _{\|\mathbf{u}\| \rightarrow \infty} \frac{f^{i}(\mathbf{u})}{\varphi(\|\mathbf{u}\|)},
$$

for all $\mathbf{u} \in \mathbb{R}_{+}^{N}$ and $i=1, \ldots, N$.

Among the variety of works mentioned above, we are interested in the following result.

Res $A$. Problem $(P)$ has at least one positive solution if either $\mathbf{f}_{0}=0, \mathbf{f}_{\infty}=\infty$ or $\mathbf{f}_{0}=\infty, \mathbf{f}_{\infty}=0$.

Wang [10] proved Res A when each $h_{i}:[0,1] \rightarrow \mathbb{R}_{+}$ is continuous and $\varphi$ satisfies that there exist two increasing homeomorphisms $\psi_{1}$ and $\psi_{2}$ of $(0, \infty)$ onto $(0, \infty)$ such that

$$
\psi_{1}(\sigma) \varphi(x) \leq \varphi(\sigma x) \leq \psi_{2}(\sigma) \varphi(x), \quad \text { for } \sigma, x>0
$$

Do Ó et al. [7] also proved Res A when $\varphi=\varphi_{p}$ and each $h_{i} \in \mathscr{H}_{\varphi_{p}}\left(=\mathscr{H}_{\psi}\right)$.

The aim of this paper is to prove Res A when $\varphi$ satisfies condition (A) and each $h_{i} \in \mathscr{H}_{\psi}$. More precisely, we state our main theorem as follows.

Theorem 2. Assume (A), $(H)$, and $(F)$ hold. Then problem $(P)$ satisfies Res $A$.

Extension of results in [10] or [7] to Theorem 2 is not obvious mainly due to the singularity of $h_{i}$ in comparison with Wang and lack of homogeneity of the general operator $\varphi$ in comparison with Do et al.

For proofs, we introduce a newly developed solution operator for $(P)$ motivated by Sim and Lee [15]. And then we make use of the fixed point theorem of a cone for the existence of positive solutions.

This paper is organized as follows. In Section 2, we introduce a solution operator for problem $(P)$ and prove the compactness of the operator. In Section 3, we prove our main theorem.

\section{A Solution Operator}

Let us consider a simple scalar problem of the form

$$
\begin{gathered}
-\varphi\left(w^{\prime}\right)^{\prime}=g(t), \quad t \in(0,1), \\
w(0)=w(1)=0,
\end{gathered}
$$

where $\varphi$ satisfies (A) and $g \geq 0$ with $g \in \mathscr{H}_{\varphi}$.

Since $g$ may not be in $L^{1}(0,1)$ as we see the example in the introduction section, in this case, the solution of $(W)+$ (D) may not be in $C^{1}[0,1]$. So by a solution to this problem, we understand a function $w \in C_{0}[0,1] \cap C^{1}(0,1)$ with $\varphi\left(w^{\prime}\right)$ absolutely continuous which satisfies $(W)$.

We first give some remarks for calculations later on.

Remark 3. From condition (A), we get

$$
\begin{gathered}
\sigma x \leq \varphi^{-1}[\gamma(\sigma) \varphi(x)] \\
\varphi^{-1}[\sigma \varphi(x)] \leq \psi^{-1}(\sigma) x, \quad \text { for } \sigma, x>0 .
\end{gathered}
$$

Remark 4. Let $h \in L_{\text {loc }}^{1}\left((0,1), \mathbb{R}_{+}\right)$. Then for any fixed $s \in$ $(0,1 / 2)$, we know $\int_{s}^{1 / 2} h(\tau) d \tau<\infty$. Applying $\sigma=\int_{s}^{1 / 2} h(\tau) d \tau$ and $x=\varphi^{-1}(1)$ in Remark 3, we get

$$
\varphi^{-1}\left(\int_{s}^{1 / 2} h(\tau) d \tau\right) \leq \varphi^{-1}(1) \psi^{-1}\left(\int_{s}^{1 / 2} h(\tau) d \tau\right)
$$

This implies $\mathscr{H}_{\psi} \subset \mathscr{H}_{\varphi}$. 
Remark 5. If $h \in \mathscr{H}_{\varphi}$, then, for any fixed $\sigma \in(0,1)$,

$$
\begin{aligned}
& \varphi^{-1}\left(\int_{s}^{\sigma} h(\tau) d \tau\right) \in L^{1}\left(0, \frac{1}{2}\right], \\
& \varphi^{-1}\left(\int_{\sigma}^{s} h(\tau) d \tau\right) \in L^{1}\left[\frac{1}{2}, 1\right) .
\end{aligned}
$$

We need a lemma which guarantees concavity of solutions. The proof is similar to Lemma 2.3 in Wang [10].

Lemma 6. Let $w \in C_{0}[0,1] \cap C^{1}(0,1)$ satisfy $\varphi\left(w^{\prime}\right)^{\prime} \leq 0$ on $(0,1)$. Then $w$ is concave on $[0,1]$ and $\min _{t \in[1 / 4,3 / 4]} w(t) \geq$ $(1 / 4)\|w\|_{\infty}$, where $\|w\|_{\infty}$ is the supremum norm of $w$.

Let $w$ be a solution of $(W)+(D)$.

Then integrating both sides of $(W)$ on the interval $[s, 1 / 2]$ for $s \in(0,1 / 2]$ and $[1 / 2, s]$ for $s \in[1 / 2,1)$, respectively, we find that $(W)+(D)$ is equivalent to

$$
\begin{aligned}
& w^{\prime}(s)=\varphi^{-1}\left(a+\int_{s}^{1 / 2} g(\tau) d \tau\right), \quad w(0)=0, s \in\left(0, \frac{1}{2}\right], \\
& w^{\prime}(s)=\varphi^{-1}\left(-a+\int_{1 / 2}^{s} g(\tau) d \tau\right), \quad w(1)=0, s \in\left[\frac{1}{2}, 1\right),
\end{aligned}
$$

where $a=\varphi\left(w^{\prime}(1 / 2)\right)$. We show that $\varphi^{-1}\left(a+\int_{s}^{1 / 2} g(\tau) d \tau\right) \epsilon$ $L^{1}(0,1 / 2]$. Indeed, by Lemma 6 , solution $w$ has a unique maximal point. That is, there exists a unique $\sigma_{w} \in(0,1)$ such that $w\left(\sigma_{w}\right)=\max _{t \in[0,1]} w(t)$. Since $w^{\prime}\left(\sigma_{w}\right)=0$, we see from (13) that

$$
\varphi^{-1}\left(a+\int_{\sigma_{w}}^{1 / 2} g(\tau) d \tau\right)=0
$$

Since $\varphi$ is an odd homeomorphism, $a+\int_{\sigma_{w}}^{1 / 2} g(\tau) d \tau=0$, and by Remark 5, we get

$$
\begin{gathered}
\varphi^{-1}\left(a+\int_{s}^{1 / 2} g(\tau) d \tau\right) \\
=\varphi^{-1}\left(-\int_{\sigma_{w}}^{1 / 2} g(\tau) d \tau+\int_{s}^{1 / 2} g(\tau) d \tau\right) \\
=\varphi^{-1}\left(\int_{s}^{\sigma} h(\tau) d \tau\right) \in L^{1}\left(0, \frac{1}{2}\right] .
\end{gathered}
$$

Similar argument shows that $\varphi^{-1}\left(-a+\int_{1 / 2}^{s} g(\tau) d \tau\right) \quad \epsilon$ $L^{1}[1 / 2,1)$. Now we integrate both sides of (13) on the interval $[0, t]$ for $t \in[0,1 / 2]$ and on the interval $[t, 1]$ for $t \in[1 / 2,1]$, respectively. Then we get

$$
w(t)= \begin{cases}\int_{0}^{t} \varphi^{-1}\left(a+\int_{s}^{1 / 2} g(\tau) d \tau\right) d s, & t \in\left[0, \frac{1}{2}\right], \\ \int_{t}^{1} \varphi^{-1}\left(-a+\int_{1 / 2}^{s} g(\tau) d \tau\right) d s, & t \in\left[\frac{1}{2}, 1\right] .\end{cases}
$$

Let us check $w(1 / 2)^{-}=w(1 / 2)^{+}$. For $a \in \mathbb{R}$, define

$$
\begin{aligned}
G(a)= & \int_{0}^{1 / 2} \varphi^{-1}\left(a+\int_{s}^{1 / 2} g(\tau) d \tau\right) d s \\
& -\int_{1 / 2}^{1} \varphi^{-1}\left(-a+\int_{1 / 2}^{s} g(\tau) d \tau\right) d s .
\end{aligned}
$$

Then the function $G: \mathbb{R} \rightarrow \mathbb{R}$ is well-defined. If $G$ has a unique zero, then $w(1 / 2)^{-}=w(1 / 2)^{+}$. For this, we give the following lemma. The proof generally follows the lines of proof of Lemma 2.2 in Sim and Lee [15].

Lemma 7. For given $g \in \mathscr{H}_{\varphi}$, the function $G$ defined in (17) has a unique zero $a=a(g)$ in $\mathbb{R}$.

Consequently, if $\varphi$ satisfies (A) and $g \in \mathscr{H}_{\varphi}$, then the solution $w$ of $(W)+(D)$ can be represented by

$$
w(t)= \begin{cases}\int_{0}^{t} \varphi^{-1}\left(a(g)+\int_{s}^{1 / 2} g(\tau) d \tau\right) d s, & t \in\left[0, \frac{1}{2}\right], \\ \int_{t}^{1} \varphi^{-1}\left(-a(g)+\int_{1 / 2}^{s} g(\tau) d \tau\right) d s, & t \in\left[\frac{1}{2}, 1\right],\end{cases}
$$

where $a(g) \in \mathbb{R}$ uniquely satisfies

$$
\begin{aligned}
\int_{0}^{1 / 2} & \varphi^{-1}\left(a(g)+\int_{s}^{1 / 2} g(\tau) d \tau\right) d s \\
& =\int_{1 / 2}^{1} \varphi^{-1}\left(-a(g)+\int_{1 / 2}^{s} g(\tau) d \tau\right) d s .
\end{aligned}
$$

On the other hand, it is not hard to see that a function $w$ defined in (18) satisfies $w \in C_{0}[0,1] \cap C^{1}(0,1)$, and $\varphi\left(w^{\prime}\right)$ is absolutely continuous on $(0,1)$ and $w$ is in turn a solution of $(W)+(D)$

Now we come back to our main problem

$$
\begin{aligned}
-\varphi\left(u_{1}^{\prime}\right)^{\prime} & =h_{1}(t) f^{1}(\mathbf{u}), \\
\vdots & \\
-\varphi\left(u_{N}^{\prime}\right)^{\prime} & =h_{N}(t) f^{N}(\mathbf{u}), \quad t \in(0,1), \\
u_{i}(0) & =0=u_{i}(1), \quad i=1, \ldots, N .
\end{aligned}
$$

We finally introduce the corresponding solution operator for $\left(P^{\prime}\right)$ and prove compactness of the operator. For this purpose, we need a preliminary lemma. 
Lemma 8. If $h \in \mathscr{H}_{\psi}$, then, for given $\alpha \in C[0,1], \alpha h \in \mathscr{H}_{\varphi}$.

Proof. Let $h \in \mathscr{H}_{\psi}$ and $\alpha \in C[0,1]$ be given. Then applying Remark 3 with $\sigma=\int_{s}^{1 / 2} h(\tau) d \tau, x=\varphi^{-1}\left(\|\alpha\|_{\infty}\right)$ and using the fact $h \in \mathscr{H}_{\psi}$, we get

$$
\begin{aligned}
\int_{0}^{1 / 2} & \varphi^{-1}\left(\int_{s}^{1 / 2} \alpha(\tau) h(\tau) d \tau\right) d s \\
& \leq \int_{0}^{1 / 2} \varphi^{-1}\left(\|\alpha\|_{\infty} \int_{s}^{1 / 2} h(\tau) d \tau\right) d s \\
& \leq \varphi^{-1}\left(\|\alpha\|_{\infty}\right) \int_{0}^{1 / 2} \psi^{-1}\left(\int_{s}^{1 / 2} h(\tau) d \tau\right) d s<\infty
\end{aligned}
$$

Similarly, we can prove

$$
\int_{1 / 2}^{1} \varphi^{-1}\left(\int_{1 / 2}^{s} \alpha(\tau) h(\tau) d \tau\right) d s<\infty
$$

This lemma should be more natural if it is valid under assumption $h \in \mathscr{H}_{\varphi}$. Even though it is true for the case $\varphi=\varphi_{p}$, the $p$-Laplace operator, it seems not easy to prove in general mainly caused by lack of homogeneity of $\varphi$.

To set up the solution operator for $\left(P^{\prime}\right)$, let us define $E$ as the Banach space $\underbrace{C_{0}[0,1] \times \cdots \times C_{0}[0,1]}_{N}$ with norm $\|\mathbf{u}\|_{\infty}=$ $\Sigma_{i=1}^{N}\left\|u_{i}\right\|_{\infty}$ and define a cone $K$ by taking

$$
K=\left\{\mathbf{u} \in E \mid u_{i} \text { is concave on }[0,1], i=1, \ldots, N\right\} \text {. }
$$

Let $\mathbf{u} \in K$ and $h_{i} \in \mathscr{H}_{\psi}, i=1, \ldots, N$; then $f^{i}(\mathbf{u}) \in C[0,1]$ and by Lemma $8, h_{i} f^{i}(\mathbf{u}) \in \mathscr{H}_{\varphi}$. Let us apply the solution representation for $(W)+(D)$ replacing $g$ with $h_{i} f^{i}(\mathbf{u})$; then we get

$$
u_{i}(t)=\left\{\begin{aligned}
& \int_{0}^{t} \varphi^{-1}\left(a^{i}\left(h_{i} f^{i}(\mathbf{u})\right)\right. \\
&\left.+\int_{s}^{1 / 2} h_{i}(\tau) f^{i}(\mathbf{u}(\tau)) d \tau\right) d s, \\
& 0 \leq t \leq \frac{1}{2}, \\
& \int_{t}^{1} \varphi^{-1}\left(-a^{i}\left(h_{i} f^{i}(\mathbf{u})\right)\right. \\
&\left.\quad+\int_{1 / 2}^{s} h_{i}(\tau) f^{i}(\mathbf{u}(\tau)) d \tau\right) d s, \\
& \frac{1}{2} \leq t \leq 1,
\end{aligned}\right.
$$

where $a^{i}\left(h_{i} f^{i}(\mathbf{u})\right)$ is a unique zero of

$$
\begin{aligned}
& \int_{0}^{1 / 2} \varphi^{-1}\left(a^{i}\left(h_{i} f^{i}(\mathbf{u})\right)+\int_{s}^{1 / 2} h_{i}(\tau) f^{i}(\mathbf{u}(\tau)) d \tau\right) d s \\
& \quad=\int_{1 / 2}^{1} \varphi^{-1}\left(-a^{i}\left(h_{i} f^{i}(\mathbf{u})\right)+\int_{1 / 2}^{s} h_{i}(\tau) f^{i}(\mathbf{u}(\tau)) d \tau\right) d s
\end{aligned}
$$

Now for $\mathbf{u} \in K$, let us define

$$
T^{i}(\mathbf{u})(t)=\left\{\begin{aligned}
\int_{0}^{t} \varphi^{-1}\left(a^{i}\left(h_{i} f^{i}(\mathbf{u})\right)\right. \\
\left.\quad+\int_{s}^{1 / 2} h_{i}(\tau) f^{i}(\mathbf{u}(\tau)) d \tau\right) d s, \\
t \in\left[0, \frac{1}{2}\right], \\
\int_{t}^{1} \varphi^{-1}\left(-a^{i}\left(h_{i} f^{i}(\mathbf{u})\right)\right. \\
\left.\quad+\int_{1 / 2}^{s} h_{i}(\tau) f^{i}(\mathbf{u}(\tau)) d \tau\right) d s, \\
t \in\left[\frac{1}{2}, 1\right], \\
T(\mathbf{u})=\left(T^{1}(\mathbf{u}), \ldots, T^{N}(\mathbf{u})\right) .
\end{aligned}\right.
$$

Then by Lemma $6, T(K) \subset K$ and we see that $\mathbf{u}$ is a positive solution of $\left(P^{\prime}\right)$ if and only if $\mathbf{u}=T(\mathbf{u})$ on $K$.

We finally prove the solution operator $T: K \rightarrow K$ is completely continuous. For this, we need a couple of lemmas about the properties of $a^{i}\left(h_{i} f^{i}(\mathbf{u})\right)$. Since $h_{i}$ and $f^{i}$ are fixed, we regard $a^{i}\left(h_{i} f^{i}(\mathbf{u})\right)$ as a function of $\mathbf{u} \in K$. The proofs of the following two lemmas are mainly induced by the monotonicity of $\varphi$ and similar to proofs of Lemmas 3.1 and 3.2 in Sim and Lee [15].

Lemma 9. $a^{i}$ sends bounded sets in $K$ into bounded sets in $\mathbb{R}$ for $i=1, \ldots, N$.

Lemma 10. $a^{i}: K \rightarrow \mathbb{R}$ is continuous for $i=1, \ldots, N$.

Lemma 11. $T: K \rightarrow K$ is completely continuous.

Proof. Continuity of $T^{i}$ can be done by using the Lebesgue Dominated Convergence Theorem with aid of the continuity of $a^{i}$. Let $B$ be a bounded subset of $K$. Then it is enough to prove $T^{i}(B)$ is uniformly bounded and equicontinuous. We first prove that $T^{i}(B)$ is uniformly bounded. Indeed, take $M_{B}=\sup \left\{\left\|f^{i}(\mathbf{u})\right\|_{\infty} \mid \mathbf{u} \in B\right\}, K_{i}\left(=K_{i}\left(h_{i}, M_{B}\right)\right)=$ $\sup \left\{\left|a^{i}\left(h_{i} f^{i}(\mathbf{u})\right)\right| \quad \mid \mathbf{u} \in B\right\}$, and denote simply $a_{\mathbf{u}}^{i} \triangleq$ $a^{i}\left(h_{i} f^{i}(\mathbf{u})\right)$. We compute the bound on the interval $(0,1 / 2]$; 
the bound on the interval $[1 / 2,1)$ can be obtained by the similar way. Consider

$$
\begin{aligned}
& \left|T^{i}(\mathbf{u})(t)\right| \\
& \quad \leq \int_{0}^{t} \varphi^{-1}\left(\left|a_{\mathbf{u}}^{i}\right|+\int_{s}^{1 / 2} h_{i}(\tau) f^{i}(\mathbf{u}(\tau)) d \tau\right) d s \\
& \quad \leq \int_{0}^{1 / 2} \varphi^{-1}\left(K_{i}+M_{B} \int_{s}^{1 / 2} h_{i}(\tau) d \tau\right) d s .
\end{aligned}
$$

Case $1\left(h_{i} \in L^{1}(0,1 / 2]\right)$

$$
\begin{aligned}
\left|T^{i}(\mathbf{u})(t)\right| & \leq \int_{0}^{1 / 2} \varphi^{-1}\left(K_{i}+M_{B} \int_{0}^{1 / 2} h_{i}(\tau) d \tau\right) d s \\
& =\frac{1}{2} \varphi^{-1}\left(K_{i}+M_{B}\left\|h_{i}\right\|_{L^{1}(0,1 / 2]}\right) .
\end{aligned}
$$

Case $2\left(h_{i} \notin L^{1}(0,1 / 2]\right)$. Let $H(s)=\int_{s}^{1 / 2} h_{i}(\tau) d \tau$; then $h_{i} \in$ $L_{\text {loc }}^{1}(0,1)$ implies that $H$ is continuous on $(0,1 / 2], H(s)<\infty$ for $s \in(0,1 / 2]$ and $H\left(0^{+}\right)=\infty$. Thus we may choose $s_{*} \in$ $(0,1 / 2)$ satisfying

$$
\frac{K_{i}}{M_{B}}=H\left(s_{*}\right)\left(=\int_{s_{*}}^{1 / 2} h_{i}(\tau) d \tau\right) .
$$

If $s \leq s_{*}$, then

$$
\begin{aligned}
\int_{0}^{s_{*}} & \varphi^{-1}\left(K_{i}+M_{B} \int_{s}^{1 / 2} h_{i}(\tau) d \tau\right) d s \\
& =\int_{0}^{s_{*}} \varphi^{-1}\left(M_{B}\left(\int_{s_{*}}^{1 / 2} h_{i}(\tau) d \tau+\int_{s}^{1 / 2} h_{i}(\tau) d \tau\right)\right) d s \\
& \leq \int_{0}^{s_{*}} \varphi^{-1}\left(2 M_{B} \int_{s}^{1 / 2} h_{i}(\tau) d \tau\right) d s
\end{aligned}
$$

On the other hand, if $s>s_{*}$, then

$$
\begin{aligned}
\int_{s_{*}}^{1 / 2} & \varphi^{-1}\left(K_{i}+M_{B} \int_{s}^{1 / 2} h_{i}(\tau) d \tau\right) d s \\
& \leq \int_{s_{*}}^{1 / 2} \varphi^{-1}\left(K_{i}+M_{B} \int_{s_{*}}^{1 / 2} h_{i}(\tau) d \tau\right) d s \\
& \leq \int_{s_{*}}^{1 / 2} \varphi^{-1}\left(2 M_{B} \int_{s_{*}}^{1 / 2} h_{i}(\tau) d \tau\right) d s .
\end{aligned}
$$

Applying Remark 3 with $\sigma=\int_{s}^{1 / 2} h_{i}(\tau) d \tau$ and $x=\varphi^{-1}\left(2 M_{B}\right)$, we get

$$
\begin{aligned}
\left|T^{i}(\mathbf{u})(t)\right| & \\
\leq & \int_{0}^{s_{*}} \varphi^{-1}\left(K_{i}+M_{B} \int_{s}^{1 / 2} h_{i}(\tau) d \tau\right) d s \\
& +\int_{s_{*}}^{1 / 2} \varphi^{-1}\left(K_{i}+M_{B} \int_{s}^{1 / 2} h_{i}(\tau) d \tau\right) d s \\
\leq & \int_{0}^{s_{*}} \varphi^{-1}\left(2 M_{B} \int_{s}^{1 / 2} h_{i}(\tau) d \tau\right) d s \\
& +\int_{s_{*}}^{1 / 2} \varphi^{-1}\left(2 M_{B} \int_{s_{*}}^{1 / 2} h_{i}(\tau) d \tau\right) d s \\
\leq & \varphi^{-1}\left(2 M_{B}\right) \int_{0}^{s_{*}} \psi^{-1}\left(\int_{s}^{1 / 2} h_{i}(\tau) d \tau\right) d s \\
& +\left(\frac{1}{2}-s_{*}\right) \varphi^{-1}\left(2 M_{B}\left\|h_{i}\right\|_{L^{1}\left(s_{*}, 1 / 2\right]}\right) .
\end{aligned}
$$

By the fact $h_{i} \in \mathscr{H}_{\psi}$, all bounds above are finite and independent on $\mathbf{u} \in B$ and $t \in[0,1 / 2]$. Thus $T^{i}(B)$ is uniformly bounded. $t_{2}$.

We finally prove the equicontinuity of $T^{i}(B)$. Assume $t_{1}<$

Case $1\left(t_{1}, t_{2} \in[0,1 / 2]\right)$

$$
\begin{aligned}
& \mid T^{i}(\mathbf{u})\left(t_{1}\right)-T^{i}(\mathbf{u})\left(t_{2}\right) \mid \\
& \quad \leq \int_{t_{1}}^{t_{2}} \varphi^{-1}\left(\left|a_{\mathbf{u}}^{i}\right|+\int_{s}^{1 / 2} h_{i}(\tau) f^{i}(\mathbf{u}(\tau)) d \tau\right) d s \\
& \quad \leq \int_{t_{1}}^{t_{2}} \varphi^{-1}\left(K_{i}+M_{B} \int_{s}^{1 / 2} h_{i}(\tau) d \tau\right) d s
\end{aligned}
$$

Let $h_{i} \in L^{1}(0,1 / 2]$; then we can easily see

$$
\begin{aligned}
& \left|T^{i}(\mathbf{u})\left(t_{1}\right)-T^{i}(\mathbf{u})\left(t_{2}\right)\right| \\
& \quad \leq \varphi^{-1}\left(K_{i}+M_{B}\left\|h_{i}\right\|_{L^{1}(0,1 / 2]}\right)\left|t_{1}-t_{2}\right| .
\end{aligned}
$$

Let $h_{i} \notin L^{1}(0,1 / 2]$; then, for $s_{*} \in(0,1 / 2)$ defined in (28),

$$
\begin{aligned}
& \left|T^{i}(\mathbf{u})\left(t_{1}\right)-T^{i}(\mathbf{u})\left(t_{2}\right)\right| \\
& \quad \leq \int_{t_{1}}^{t_{2}} \varphi^{-1}\left(M_{B}\left(\int_{s_{*}}^{1 / 2} h_{i}(\tau) d \tau+\int_{s}^{1 / 2} h_{i}(\tau) d \tau\right)\right) d s .
\end{aligned}
$$


Subcase $1\left(0 \leq t_{1}<t_{2} \leq s_{*}\right)$. Applying Remark 3 with $\sigma=$ $\int_{s}^{1 / 2} h_{i}(\tau) d \tau$ and $x=\varphi^{-1}\left(2 M_{B}\right)$, we get

$$
\begin{aligned}
&\left|T^{i}(\mathbf{u})\left(t_{1}\right)-T^{i}(\mathbf{u})\left(t_{2}\right)\right| \\
& \quad \leq \int_{t_{1}}^{t_{2}} \varphi^{-1}\left(2 M_{B} \int_{s}^{1 / 2} h_{i}(\tau) d \tau\right) d s \\
& \quad \leq \varphi^{-1}\left(2 M_{B}\right) \int_{t_{1}}^{t_{2}} \psi^{-1}\left(\int_{s}^{1 / 2} h_{i}(\tau) d \tau\right) d s .
\end{aligned}
$$

Subcase $2\left(s_{*} \leq t_{1}<t_{2}\right)$

$$
\begin{aligned}
& \left|T^{i}(\mathbf{u})\left(t_{1}\right)-T^{i}(\mathbf{u})\left(t_{2}\right)\right| \\
& \quad \leq \int_{t_{1}}^{t_{2}} \varphi^{-1}\left(2 M_{B} \int_{s_{*}}^{1 / 2} h_{i}(\tau) d \tau\right) d s \\
& \quad \leq \varphi^{-1}\left(2 M_{B}\left\|h_{i}\right\|_{L^{1}\left[s_{*}, 1 / 2\right]}\right)\left|t_{1}-t_{2}\right| .
\end{aligned}
$$

Subcase $3\left(0 \leq t_{1} \leq s_{*}<t_{2}\right)$. Consider

$$
\begin{aligned}
\mid T^{i}(\mathbf{u}) & \left(t_{1}\right)-T^{i}(\mathbf{u})\left(t_{2}\right) \mid \\
\leq & \int_{t_{1}}^{s_{*}} \varphi^{-1}\left(M_{B}\left(\int_{s_{*}}^{1 / 2} h_{i}(\tau) d \tau+\int_{s}^{1 / 2} h_{i}(\tau) d \tau\right)\right) d s \\
& +\int_{s_{*}}^{t_{2}} \varphi^{-1}\left(M_{B}\left(\int_{s_{*}}^{1 / 2} h_{i}(\tau) d \tau+\int_{s}^{1 / 2} h_{i}(\tau) d \tau\right)\right) d s \\
\leq & \int_{t_{1}}^{s_{*}} \varphi^{-1}\left(2 M_{B} \int_{s}^{1 / 2} h_{i}(\tau) d \tau\right) d s \\
& +\int_{s_{*}}^{t_{2}} \varphi^{-1}\left(2 M_{B} \int_{s_{*}}^{1 / 2} h_{i}(\tau) d \tau\right) d s \\
\leq & \varphi^{-1}\left(2 M_{B}\right) \int_{t_{1}}^{t_{2}} \psi^{-1}\left(\int_{s}^{1 / 2} h_{i}(\tau) d \tau\right) d s \\
& +\varphi^{-1}\left(2 M_{B}\left\|h_{i}\right\|_{L^{1}\left[s_{*}, 1 / 2\right]}\right)\left|t_{1}-t_{2}\right| .
\end{aligned}
$$

Bounds of all cases above are independent of $\mathbf{u} \in B$ and by the fact $h_{i} \in \mathscr{H}_{\psi}$, we see that each bound converges to 0 as $\left|t_{1}-t_{2}\right| \rightarrow 0$.

Case $2\left(t_{1}, t_{2} \in[1 / 2,1]\right)$. Proof can be done by the same argument as Case 1.

Case $3\left(0<t_{1} \leq 1 / 2<t_{2}<1\right)$. Without loss of generality, we assume $1 / 4 \leq t_{1} \leq 1 / 2<t_{2} \leq 3 / 4$. Then, by using the definition of $a_{\mathbf{u}}^{i}$, we obtain

$$
\begin{aligned}
\mid T^{i}(\mathbf{u}) & \left(t_{1}\right)-T^{i}(\mathbf{u})\left(t_{2}\right) \mid \\
= & \mid \int_{0}^{t_{1}} \varphi^{-1}\left(a_{\mathbf{u}}^{i}+\int_{s}^{1 / 2} h_{i}(\tau) f^{i}(\mathbf{u}(\tau)) d \tau\right) d s \\
& -\int_{t_{2}}^{1} \varphi^{-1}\left(-a_{\mathbf{u}}^{i}+\int_{1 / 2}^{s} h_{i}(\tau) f^{i}(\mathbf{u}(\tau)) d \tau\right) d s \mid
\end{aligned}
$$

$$
\begin{aligned}
= & \mid \int_{0}^{t_{1}} \varphi^{-1}\left(a_{\mathbf{u}}^{i}+\int_{s}^{1 / 2} h_{i}(\tau) f^{i}(\mathbf{u}(\tau)) d \tau\right) d s \\
& -\int_{0}^{1 / 2} \varphi^{-1}\left(a_{\mathbf{u}}^{i}+\int_{s}^{1 / 2} h_{i}(\tau) f^{i}(\mathbf{u}(\tau)) d \tau\right) d s \\
& +\int_{1 / 2}^{1} \varphi^{-1}\left(-a_{\mathbf{u}}^{i}+\int_{1 / 2}^{s} h_{i}(\tau) f^{i}(\mathbf{u}(\tau)) d \tau\right) d s \\
& -\int_{t_{2}}^{1} \varphi^{-1}\left(-a_{\mathbf{u}}^{i}+\int_{1 / 2}^{s} h_{i}(\tau) f^{i}(\mathbf{u}(\tau)) d \tau\right) d s \mid \\
\leq & \int_{t_{1}}^{1 / 2} \varphi^{-1}\left(K_{i}+M_{B} \int_{s}^{1 / 2} h_{i}(\tau) d \tau\right) d s \\
& +\int_{1 / 2}^{t_{2}} \varphi^{-1}\left(K_{i}+M_{B} \int_{1 / 2}^{s} h_{i}(\tau) d \tau\right) d s \\
\leq & \int_{t_{1}}^{1 / 2} \varphi^{-1}\left(K_{i}+M_{B} \int_{1 / 4}^{1 / 2} h_{i}(\tau) d \tau\right) d s \\
& +\varphi^{-1}\left(K_{i}+M_{B}\left\|h_{i}\right\|_{L^{1}[1 / 2,3 / 4]}\right)\left|t_{2}-\frac{1}{2}\right| \\
& +\int_{1 / 2}^{t_{2}} \varphi^{-1}\left(K_{i}+M_{B} \int_{1 / 2}^{3 / 4} h_{i}(\tau) d \tau\right) d s \\
= & \varphi^{-1}\left(K_{i}+M_{B}\left\|h_{i}\right\|_{L^{1}[1 / 4,1 / 2]}\right)\left|t_{1}-\frac{1}{2}\right|
\end{aligned}
$$

Conclusion is the same as Case 1 and it completes the proof of equicontinuity.

\section{Proof of Theorem 2}

In this section, we prove our main theorem. Basic tool for the proof is the following well-known fixed point theorem (see $[16,17])$.

Lemma 12. Let $E$ be a Banach space and let $K$ be a cone in $E$. Assume that $\Omega_{1}$ and $\Omega_{2}$ are open subsets of $E$ with $0 \in \Omega_{1}$, $\overline{\Omega_{1}} \subset \Omega_{2}$. Assume that $T: K \cap \overline{\Omega_{2}} \backslash \Omega_{1} \rightarrow K$ is completely continuous such that either

$\|T \mathbf{u}\| \leq\|\mathbf{u}\|, \quad$ for $\mathbf{u} \in K \cap \partial \Omega_{1} ;$

$\|T \mathbf{u}\| \geq\|\mathbf{u}\|, \quad$ for $\mathbf{u} \in K \cap \partial \Omega_{2}$

or $\quad\|T \mathbf{u}\| \geq\|\mathbf{u}\|, \quad$ for $\mathbf{u} \in K \cap \partial \Omega_{1}$;

$\|T \mathbf{u}\| \leq\|\mathbf{u}\|, \quad$ for $\mathbf{u} \in K \cap \partial \Omega_{2}$.

Then $T$ has a fixed point in $K \cap \overline{\Omega_{2}} \backslash \Omega_{1}$. 
Proof of Theorem 2. (1) Let $\mathbf{f}_{0}=0$; then $f_{0}^{i}=0, i=1, \ldots, N$. For convenience, we denote

$$
\begin{aligned}
& H_{0}^{i} \triangleq \int_{0}^{1 / 2} \psi^{-1}\left(\int_{s}^{1 / 2} h_{i}(\tau) d \tau\right) d s, \\
& H_{1}^{i} \triangleq \int_{1 / 2}^{1} \psi^{-1}\left(\int_{1 / 2}^{s} h_{i}(\tau) d \tau\right) d s,
\end{aligned}
$$

where $i=1, \ldots, N$. Then $h_{i} \in \mathscr{H}_{\psi}$ implies $H_{0}^{i}, H_{1}^{i}<\infty$. Choose $\epsilon>0$ sufficiently small so that

$$
\psi^{-1}(\epsilon) \max \left\{H_{0}^{i}, H_{1}^{i} \mid i=1, \ldots, N\right\} \leq \frac{1}{N} .
$$

Then we see that

$$
\psi^{-1}(\epsilon) \max \left\{H_{0}^{i}, H_{1}^{i}\right\} \leq \frac{1}{N}, \quad \text { for } i=1, \ldots, N
$$

Since $f_{0}^{i}=0$, there exists $r_{1}^{i}\left(=r_{1}^{i}(\epsilon)\right)>0$ such that, for $\mathbf{x} \in \mathbb{R}_{+}^{N}$ with $\|\mathbf{x}\| \leq r_{1}^{i}$,

$$
f^{i}(\mathbf{x}) \leq \varepsilon \varphi(\|\mathbf{x}\|), \quad \text { for } i=1, \ldots, N .
$$

Denote $K_{a}=\left\{\mathbf{u} \in K \mid\|\mathbf{u}\|_{\infty}<a\right\}$ for $a>0$ and take $r_{1}=\min \left\{r_{1}^{i} \mid i=1, \ldots, N\right\}$. Then since $T(\mathbf{u}) \in K$ for $\mathbf{u} \in \partial K_{r_{1}}$, there exists unique $\sigma_{i} \in(0,1)$ such that $T^{i}(\mathbf{u})\left(\sigma_{i}\right)=$ $\max _{t \in[0,1]} T^{i}(\mathbf{u})(t)$ and $T^{i}(\mathbf{u})^{\prime}\left(\sigma_{i}\right)=0$. We first consider the case $\sigma_{i} \in(0,1 / 2]$. Consider

$$
0=T^{i}(\mathbf{u})^{\prime}\left(\sigma_{i}\right)=\varphi^{-1}\left(a_{\mathbf{u}}^{i}+\int_{\sigma_{i}}^{1 / 2} h_{i}(\tau) f^{i}(\mathbf{u}(\tau)) d \tau\right) .
$$

Since $\varphi$ is an odd homeomorphism, $a_{\mathbf{u}}^{i}=-\int_{\sigma_{i}}^{1 / 2} h_{i}(\tau)$ $f^{i}(\mathbf{u}(\tau)) d \tau$. Using (43) and applying Remark 3 with $\sigma=\epsilon$, $x=\varphi^{-1}\left(\varphi\left(r_{1}\right) \int_{s}^{1 / 2} h_{i}(\tau) d \tau\right)$, and then $\sigma=\int_{s}^{1 / 2} h_{i}(\tau) d \tau, x=r_{1}$ consecutively, we obtain

$$
\begin{aligned}
\left\|T^{i}(\mathbf{u})\right\|_{\infty}= & T^{i}(\mathbf{u})\left(\sigma_{i}\right) \\
= & \int_{0}^{\sigma_{i}} \varphi^{-1}\left(a_{\mathbf{u}}^{i}+\int_{s}^{1 / 2} h_{i}(\tau) f^{i}(\mathbf{u}(\tau)) d \tau\right) d s \\
= & \int_{0}^{\sigma_{i}} \varphi^{-1}\left(-\int_{\sigma_{i}}^{1 / 2} h_{i}(\tau) f^{i}(\mathbf{u}(\tau)) d \tau\right. \\
& \left.\quad+\int_{s}^{1 / 2} h_{i}(\tau) f^{i}(\mathbf{u}(\tau)) d \tau\right) d s
\end{aligned}
$$

$$
\begin{aligned}
& =\int_{0}^{\sigma_{i}} \varphi^{-1}\left(\int_{s}^{\sigma_{i}} h_{i}(\tau) f^{i}(\mathbf{u}(\tau)) d \tau\right) d s \\
& \leq \int_{0}^{1 / 2} \varphi^{-1}\left(\int_{s}^{1 / 2} h_{i}(\tau) f^{i}(\mathbf{u}(\tau)) d \tau\right) d s \\
& \leq \int_{0}^{1 / 2} \varphi^{-1}\left(\epsilon \varphi\left(r_{1}\right) \int_{s}^{1 / 2} h_{i}(\tau) d \tau\right) d s \\
& \leq \psi^{-1}(\epsilon) \int_{0}^{1 / 2} \varphi^{-1}\left(\varphi\left(r_{1}\right) \int_{s}^{1 / 2} h_{i}(\tau) d \tau\right) d s \\
& \leq \psi^{-1}(\epsilon)\left[\int_{0}^{1 / 2} \psi^{-1}\left(\int_{s}^{1 / 2} h_{i}(\tau) d \tau\right) d s\right] r_{1} \\
& =\psi^{-1}(\epsilon) H_{0}^{i} r_{1} .
\end{aligned}
$$

Similarly for the case $\sigma_{i} \in[1 / 2,1)$, we get

$$
\left\|T^{i}(\mathbf{u})\right\|_{\infty} \leq \psi^{-1}(\epsilon) H_{1}^{i} r_{1}
$$

Therefore combining the above two inequalities and using the definition of $\epsilon$, we get

$$
\begin{array}{r}
\left\|T^{i}(\mathbf{u})\right\|_{\infty} \leq \psi^{-1}(\epsilon) \max \left\{H_{0}^{i}, H_{1}^{i}\right\} r_{1} \leq \frac{r_{1}}{N}, \\
\text { for } i=1, \ldots, N,
\end{array}
$$

and thus

$$
\|T(\mathbf{u})\|_{\infty}=\sum_{i=1}^{N}\left\|T^{i}(\mathbf{u})\right\|_{\infty} \leq\|\mathbf{u}\|_{\infty}, \quad \text { for } \mathbf{u} \in \partial K_{r_{1}} .
$$

We now use the assumption $\mathbf{f}_{\infty}=\infty$. In this case, we may choose an index $i_{0}$ satisfying $f_{\infty}^{i_{0}}=\infty$. Take

$$
M=\frac{\gamma(32)}{\min \left\{\int_{1 / 4}^{1 / 2} h_{i_{0}}(\tau) d \tau, \int_{1 / 2}^{3 / 4} h_{i_{0}}(\tau) d \tau\right\}}>0,
$$

where $\gamma$ is the function appeared in condition (A). Then there exists $R_{M}>0$ such that, for $\mathbf{x} \in \mathbb{R}_{+}^{N}$ with $\|\mathbf{x}\| \geq R_{M}$, we have

$$
f^{i_{0}}(\mathbf{x}) \geq M \varphi(\|\mathbf{x}\|) .
$$

If $\mathbf{u} \in K$ with $\|\mathbf{u}\|_{\infty} \geq 4 R_{M}$, then by Lemma 6 , for $t \in$ $[1 / 4,3 / 4]$,

$$
\begin{gathered}
\|\mathbf{u}(t)\|=\sum_{i=1}^{N} u_{i}(t) \geq \min _{t \in[1 / 4,3 / 4]_{i=1}} \sum_{i=1}^{N} u_{i}(t) \geq \frac{1}{4}\|\mathbf{u}\|_{\infty} \geq R_{M}, \\
f^{i_{0}}(\mathbf{u}(t)) \geq M \varphi(\|\mathbf{u}(t)\|) \geq M \varphi\left(\frac{1}{4}\|\mathbf{u}\|_{\infty}\right) .
\end{gathered}
$$


Take $r_{2}>\max \left\{r_{1}, 4 R_{M}\right\}$. Then for $\mathbf{u} \in \partial K_{r_{2}}$, we get

$$
\begin{aligned}
2 T^{i_{0}}(\mathbf{u}) & \left(\frac{1}{2}\right) \\
= & \int_{0}^{1 / 2} \varphi^{-1}\left(a_{\mathbf{u}}^{i_{0}}+\int_{s}^{1 / 2} h_{i_{0}}(\tau) f^{i_{0}}(\mathbf{u}(\tau)) d \tau\right) d s \\
& +\int_{1 / 2}^{1} \varphi^{-1}\left(-a_{\mathbf{u}}^{i_{0}}+\int_{1 / 2}^{s} h_{i_{0}}(\tau) f^{i_{0}}(\mathbf{u}(\tau)) d \tau\right) d s .
\end{aligned}
$$

If $a_{\mathbf{u}}^{i_{0}} \geq 0$, then

$$
\begin{aligned}
\int_{0}^{1 / 2} & \varphi^{-1}\left(a_{\mathbf{u}}^{i_{0}}+\int_{s}^{1 / 2} h_{i_{0}}(\tau) f^{i_{0}}(\mathbf{u}(\tau)) d \tau\right) d s \\
& \geq \int_{0}^{1 / 2} \varphi^{-1}\left(\int_{s}^{1 / 2} h_{i_{0}}(\tau) f^{i_{0}}(\mathbf{u}(\tau)) d \tau\right) d s
\end{aligned}
$$

and by the definition of $a_{\mathbf{u}}^{i_{0}}$,

$$
\begin{aligned}
\int_{1 / 2}^{1} \varphi^{-1}\left(-a_{\mathbf{u}}^{i_{0}}+\int_{1 / 2}^{s} h_{i_{0}}(\tau) f^{i_{0}}(\mathbf{u}(\tau)) d \tau\right) d s \\
\quad=\int_{0}^{1 / 2} \varphi^{-1}\left(a_{\mathbf{u}}^{i_{0}}+\int_{s}^{1 / 2} h_{i_{0}}(\tau) f^{i_{0}}(\mathbf{u}(\tau)) d \tau\right) d s \geq 0 .
\end{aligned}
$$

Thus

$$
2 T^{i_{0}}(\mathbf{u})\left(\frac{1}{2}\right) \geq \int_{0}^{1 / 2} \varphi^{-1}\left(\int_{s}^{1 / 2} h_{i_{0}}(\tau) f^{i_{0}}(\mathbf{u}(\tau)) d \tau\right) d s .
$$

If $a_{\mathbf{u}}^{i_{0}}<0$, then $-a_{\mathbf{u}}^{i_{0}}>0$ and

$$
\begin{gathered}
\int_{1 / 2}^{1} \varphi^{-1}\left(-a_{\mathbf{u}}^{i_{0}}+\int_{1 / 2}^{s} h_{i_{0}}(\tau) f^{i_{0}}(u(\tau)) d \tau\right) d s \\
\geq \int_{1 / 2}^{1} \varphi^{-1}\left(\int_{1 / 2}^{s} h_{i_{0}}(\tau) f^{i_{0}}(u(\tau)) d \tau\right) d s
\end{gathered}
$$

and by the same argument, we get

$$
2 T^{i_{0}}(\mathbf{u})\left(\frac{1}{2}\right) \geq \int_{1 / 2}^{1} \varphi^{-1}\left(\int_{1 / 2}^{s} h_{i_{0}}(\tau) f^{i_{0}}(\mathbf{u}(\tau)) d \tau\right) d s
$$

Thus by using (52), we get

$$
\begin{aligned}
& 2\left\|T^{i_{0}}(\mathbf{u})\right\|_{\infty} \\
& \quad \geq 2 T^{i_{0}}(\mathbf{u})\left(\frac{1}{2}\right)
\end{aligned}
$$

$$
\geq \min \left\{\int_{0}^{1 / 2} \varphi^{-1}\left(\int_{s}^{1 / 2} h_{i_{0}}(\tau) f^{i_{0}}(\mathbf{u}(\tau)) d \tau\right) d s\right.
$$

$$
\begin{array}{r}
\left.\int_{1 / 2}^{1} \varphi^{-1}\left(\int_{1 / 2}^{s} h_{i_{0}}(\tau) f^{i_{0}}(\mathbf{u}(\tau)) d \tau\right) d s\right\} \\
\geq \min \left\{\int_{0}^{1 / 4} \varphi^{-1}\left(\int_{s}^{1 / 2} h_{i_{0}}(\tau) f^{i_{0}}(\mathbf{u}(\tau)) d \tau\right) d s\right. \\
\left.\int_{3 / 4}^{1} \varphi^{-1}\left(\int_{1 / 2}^{s} h_{i_{0}}(\tau) f^{i_{0}}(\mathbf{u}(\tau)) d \tau\right) d s\right\}
\end{array}
$$$$
\geq \min \left\{\int_{0}^{1 / 4} \varphi^{-1}\left(\int_{1 / 4}^{1 / 2} h_{i_{0}}(\tau) f^{i_{0}}(\mathbf{u}(\tau)) d \tau\right) d s,\right.
$$

$$
\begin{gathered}
\left.\int_{3 / 4}^{1} \varphi^{-1}\left(\int_{1 / 2}^{3 / 4} h_{i_{0}}(\tau) f^{i_{0}}(\mathbf{u}(\tau)) d \tau\right) d s\right\} \\
\geq \min \left\{\int_{0}^{1 / 4} \varphi^{-1}\left(M \varphi\left(\frac{1}{4}\|\mathbf{u}\|_{\infty}\right) \int_{1 / 4}^{1 / 2} h_{i_{0}}(\tau) d \tau\right) d s,\right.
\end{gathered}
$$

$$
\begin{gathered}
\left.\int_{3 / 4}^{1} \varphi^{-1}\left(M \varphi\left(\frac{1}{4}\|\mathbf{u}\|_{\infty}\right) \int_{1 / 2}^{3 / 4} h_{i_{0}}(\tau) d \tau\right) d s\right\} \\
=\frac{1}{4} \varphi^{-1}\left(M \varphi\left(\frac{1}{4}\|\mathbf{u}\|_{\infty}\right)\right. \\
\left.\times \min \left\{\int_{1 / 4}^{1 / 2} h_{i_{0}}(\tau) d \tau, \int_{1 / 2}^{3 / 4} h_{i_{0}}(\tau) d \tau\right\}\right) .
\end{gathered}
$$

By the definition of $M$, we get

$$
2\left\|T^{i_{0}}(\mathbf{u})\right\|_{\infty} \geq \frac{1}{4} \varphi^{-1}\left(\gamma(32) \varphi\left(\frac{1}{4}\|\mathbf{u}\|_{\infty}\right)\right) .
$$

Applying Remark 3 with $\sigma=32$ and $x=(1 / 4)\|\mathbf{u}\|_{\infty}$, we get

$$
2\left\|T^{i_{0}}(\mathbf{u})\right\|_{\infty} \geq \frac{1}{4} \cdot 32 \cdot \frac{1}{4}\|\mathbf{u}\|_{\infty}=2\|\mathbf{u}\|_{\infty} .
$$

Thus

$$
\|T(\mathbf{u})\|_{\infty} \geq\left\|T^{i_{0}}(\mathbf{u})\right\|_{\infty} \geq\|\mathbf{u}\|_{\infty}, \quad \text { for } \mathbf{u} \in \partial K_{r_{2}} .
$$

Combining (48) and (62), we conclude that problem $(P)$ has at least one positive solution $\mathbf{u}$ with $r_{1} \leq\|\mathbf{u}\|_{\infty} \leq r_{2}$.

(2) We now prove the second result of Theorem 2. Let $\mathbf{f}_{0}=$ $\infty$; then there exists an index $i_{0}$ satisfying $f_{0}^{i_{0}}=\infty$. Take

$$
M=\frac{\gamma(32)}{\min \left\{\int_{1 / 4}^{1 / 2} h_{i_{0}}(\tau) d \tau, \int_{1 / 2}^{3 / 4} h_{i_{0}}(\tau) d \tau\right\}}>0 .
$$

Then there exists $r_{M}>0$ such that, for $\mathbf{x} \in \mathbb{R}_{+}^{N}$ with $\|\mathbf{x}\| \leq r_{M}$, we have

$$
f^{i_{0}}(\mathbf{x}) \geq M \varphi(\|\mathbf{x}\|) .
$$

If $\mathbf{u} \in K$ with $\|\mathbf{u}\|_{\infty} \leq r_{M}$, then by Lemma 6 , for $t \in[1 / 4,3 / 4]$,

$$
\begin{gathered}
\|\mathbf{u}(t)\| \leq\|\mathbf{u}\|_{\infty} \leq r_{M}, \\
f^{i_{0}}(\mathbf{u}(t)) \geq M \varphi(\|\mathbf{u}(t)\|) \geq M \varphi\left(\frac{1}{4}\|\mathbf{u}\|_{\infty}\right) .
\end{gathered}
$$


Take $r_{1}=r_{M}$ and let $\mathbf{u} \in \partial K_{r_{1}}$. Then

$$
\begin{aligned}
2 T^{i_{0}}(\mathbf{u}) & \left(\frac{1}{2}\right) \\
= & \int_{0}^{1 / 2} \varphi^{-1}\left(a_{\mathbf{u}}^{i_{0}}+\int_{s}^{1 / 2} h_{i_{0}}(\tau) f^{i_{0}}(\mathbf{u}(\tau)) d \tau\right) d s \\
& +\int_{1 / 2}^{1} \varphi^{-1}\left(-a_{\mathbf{u}}^{i_{0}}+\int_{1 / 2}^{s} h_{i_{0}}(\tau) f^{i_{0}}(\mathbf{u}(\tau)) d \tau\right) d s .
\end{aligned}
$$

We also consider two cases $a_{\mathbf{u}}^{i_{0}} \geq 0$ and $a_{\mathbf{u}}^{i_{0}}<0$. Applying the same argument in (1) with aid of (66), we get

$$
\begin{aligned}
& 2\left\|T^{i_{0}}(\mathbf{u})\right\|_{\infty} \\
& \geq 2 T^{i_{0}}(\mathbf{u})\left(\frac{1}{2}\right) \\
& =\frac{1}{4} \varphi^{-1}\left(M \varphi\left(\frac{1}{4}\|\mathbf{u}\|_{\infty}\right)\right. \\
& \left.\quad \times \min \left\{\int_{1 / 4}^{1 / 2} h_{i_{0}}(\tau) d \tau, \int_{1 / 2}^{3 / 4} h_{i_{0}}(\tau) d \tau\right\}\right) .
\end{aligned}
$$

By the definition of $M$, we get

$$
2\left\|T^{i_{0}}(\mathbf{u})\right\|_{\infty} \geq \frac{1}{4} \varphi^{-1}\left(\gamma(32) \varphi\left(\frac{1}{4}\|\mathbf{u}\|_{\infty}\right)\right) .
$$

Applying Remark 3 with $\sigma=32$ and $x=(1 / 4)\|\mathbf{u}\|_{\infty}$, we get

$$
2\left\|T^{i_{0}}(\mathbf{u})\right\|_{\infty} \geq \frac{1}{4} \cdot 32 \cdot \frac{1}{4}\|\mathbf{u}\|_{\infty}=2\|\mathbf{u}\|_{\infty} .
$$

Thus

$$
\|T(\mathbf{u})\|_{\infty} \geq\left\|T^{i_{0}}(\mathbf{u})\right\|_{\infty} \geq\|\mathbf{u}\|_{\infty}, \quad \text { for } \mathbf{u} \in \partial K_{r_{1}} .
$$

Let $\mathbf{f}_{\mathrm{\infty}}=0$; then $f_{\infty}^{i}=0, i=1, \ldots, N$. Define a function $\widehat{f}^{i}(t): \mathbb{R}_{+} \rightarrow \mathbb{R}_{+}$by

$$
\widehat{f}^{i}(t)=\max \left\{f^{i}(\mathbf{x}) \mid \mathbf{x} \in \mathbb{R}_{+}^{N},\|\mathbf{x}\| \leq t\right\} .
$$

By Lemma 2.8 in Wang [10], we have

$$
\widehat{f}_{\infty}^{i}=\lim _{t \rightarrow \infty} \frac{\widehat{f}^{i}(t)}{\varphi(t)}=f_{\infty}^{i}=0 .
$$

Choose $\epsilon>0$ sufficiently small so that

$$
\psi^{-1}(\epsilon) \max \left\{H_{0}^{i}, H_{1}^{i} \mid i=1, \ldots, N\right\} \leq \frac{1}{N}
$$

where $H_{0}^{i}$ and $H_{1}^{i}$ are defined as in part (1). Then we see that

$$
\psi^{-1}(\epsilon) \max \left\{H_{0}^{i}, H_{1}^{i}\right\} \leq \frac{1}{N}, \quad \text { for } i=1, \ldots, N \text {. }
$$

Since $\widehat{f}_{\infty}^{i}=0$, there exists $r_{2}^{i}\left(=r_{2}^{i}(\epsilon)\right)>$ such that, for $t \in \mathbb{R}_{+}$ with $t \geq r_{2}^{i}$,

$$
\widehat{f}^{i}(t) \leq \varepsilon \varphi(t), \quad \text { for } i=1, \ldots, N .
$$

Take $r_{2}>\max \left\{r_{1}, \max \left\{r_{2}^{i} \mid i=1, \ldots, N\right\}\right\}$. Then for $\mathbf{u} \in \partial K_{r_{2}}$, we get

$$
f^{i}(\mathbf{u}(t)) \leq \widehat{f}^{i}\left(r_{2}\right) \leq \varepsilon \varphi\left(r_{2}\right), \quad \text { for } i=1, \ldots, N .
$$

Since $T(\mathbf{u}) \in K$, there exists unique $\sigma_{i} \in(0,1)$ such that $T^{i}(\mathbf{u})\left(\sigma_{i}\right)=\max _{t \in[0,1]} T^{i}(\mathbf{u})(t)$ and $T^{i}(\mathbf{u})^{\prime}\left(\sigma_{i}\right)=0$. Considering two cases $\sigma_{i} \in(0,1 / 2]$ and $\sigma_{i} \in[1 / 2,1)$ with the same argument in (1) and using (77), we get

$$
\begin{gathered}
\left\|T^{i}(\mathbf{u})\right\|_{\infty} \leq \psi^{-1}(\epsilon) \max \left\{H_{0}^{i}, H_{1}^{i}\right\} r_{2}, \quad \text { for } i=1, \ldots, N, \\
\|T(\mathbf{u})\|_{\infty}=\sum_{i=1}^{N}\left\|T^{i}(\mathbf{u})\right\|_{\infty} \leq\|\mathbf{u}\|_{\infty}, \quad \text { for } \mathbf{u} \in \partial K_{r_{2}} .
\end{gathered}
$$

Combining (71) and (79), we conclude that problem $(P)$ has at least one positive solution $\mathbf{u}$ with $r_{1} \leq\|\mathbf{u}\|_{\infty} \leq r_{2}$ and the proof is complete.

\section{Examples}

In this section, we give some examples applicable to our main results.

Example 13. Consider the following $\varphi$-Laplacian system:

$$
\begin{gathered}
\varphi\left(u^{\prime}\right)^{\prime}+t^{-\alpha}\left[(u+v)^{p-q}+1\right]=0, \\
\varphi\left(v^{\prime}\right)^{\prime}+t^{-\beta} u^{q-1}\left(1-e^{-v}\right)=0, \quad t \in(0,1), \\
u(0)=v(0)=u(1)=v(1)=0,
\end{gathered}
$$

where $\varphi(x)=|x|^{p-2} x+|x|^{q-2} x, x \in \mathbb{R}, 1<q<p, 1<$ $\alpha, \beta<\min \{2, q\}$. We note that both $h(t)=t^{-\alpha}$ and $h(t)=t^{-\beta}$ are not in $L^{1}(0,1)$. It is easy to see that $\varphi$ is an odd increasing homeomorphism. Define functions $\psi$ and $\gamma$ given as

$$
\begin{aligned}
& \psi(\sigma)= \begin{cases}\sigma^{p-1}, & \text { if } 0<\sigma \leq 1, \\
\sigma^{q-1}, & \text { if } \sigma>1,\end{cases} \\
& \gamma(\sigma)= \begin{cases}1, & \text { if } 0<\sigma \leq 1, \\
\sigma^{p-1}, & \text { if } \sigma>1 .\end{cases}
\end{aligned}
$$

Then $\psi, \gamma:(0, \infty) \rightarrow(0, \infty)$ and $\psi$ is an increasing homeomorphism with

$$
\psi^{-1}(\sigma)= \begin{cases}\sigma^{1 /(p-1)}, & \text { if } 0<\sigma \leq 1, \\ \sigma^{1 /(q-1)}, & \text { if } \sigma>1\end{cases}
$$

If $0<\sigma \leq 1$, then $\sigma^{-(p-q)} \geq 1$ and

$$
\begin{aligned}
\frac{\varphi(\sigma x)}{\varphi(x)} & =\frac{\sigma^{p-1}\left[|x|^{p-2} x+\sigma^{-(p-q)}|x|^{q-2} x\right]}{|x|^{p-2} x+|x|^{q-2} x} \\
& \geq \sigma^{p-1}=\psi(\sigma) .
\end{aligned}
$$


If $\sigma>1$, then $\sigma^{p-q}>1$ and

$$
\begin{aligned}
\frac{\varphi(\sigma x)}{\varphi(x)} & =\frac{\sigma^{q-1}\left[\sigma^{p-q}|x|^{p-2} x+|x|^{q-2} x\right]}{|x|^{p-2} x+|x|^{q-2} x} \\
& \geq \sigma^{q-1}=\psi(\sigma) .
\end{aligned}
$$

If $0<\sigma \leq 1$, then $\sigma^{p-q} \leq 1$ and

$$
\begin{aligned}
\frac{\varphi(\sigma x)}{\varphi(x)} & =\frac{\sigma^{q-1}\left[\sigma^{p-q}|x|^{p-2} x+|x|^{q-2} x\right]}{|x|^{p-2} x+|x|^{q-2} x} \\
& \leq \sigma^{q-1} \leq 1=\gamma(\sigma) .
\end{aligned}
$$

If $\sigma>1$, then $\sigma^{-(p-q)}<1$ and

$$
\begin{aligned}
\frac{\varphi(\sigma x)}{\varphi(x)} & =\frac{\sigma^{p-1}\left[|x|^{p-2} x+\sigma^{-(p-q)}|x|^{q-2} x\right]}{|x|^{p-2} x+|x|^{q-2} x} \\
& \leq \sigma^{p-1}=\gamma(\sigma) .
\end{aligned}
$$

Thus, it follows that

$$
\psi(\sigma) \leq \frac{\varphi(\sigma x)}{\varphi(\sigma)} \leq \gamma(\sigma), \quad \forall \sigma>0, x \in \mathbb{R} .
$$

Next, we show that $h(t)=t^{-\alpha} \in \mathscr{H}_{\psi}$. Consider

$$
\begin{aligned}
\int_{s}^{1 / 2} & \tau^{-\alpha} d \tau \\
& =-\left.\frac{1}{\alpha-1} \tau^{-(\alpha-1)}\right|_{s} ^{1 / 2} \\
& =-\frac{1}{\alpha-1}\left[\left(\frac{1}{2}\right)^{-(\alpha-1)}-s^{-(\alpha-1)}\right] \\
& =\frac{1}{\alpha-1}\left[s^{-(\alpha-1)}-2^{\alpha-1}\right] \leq \frac{1}{\alpha-1} s^{-(\alpha-1)} .
\end{aligned}
$$

Since $1<\alpha<\min \{2, q\}$, then $(1 /(\alpha-1))^{1 /(\alpha-1)}>1$ and $(1 /(\alpha-1))^{1 /(\alpha-1)}>s$, for $s \in(0,1)$. Thus, $1 /(\alpha-1)>s^{\alpha-1}$, $(1 /(\alpha-1)) s^{-(\alpha-1)}>1$, and

$$
\begin{aligned}
\int_{0}^{1 / 2} & \psi^{-1}\left(\int_{s}^{1 / 2} \tau^{-\alpha} d \tau\right) d s \\
& \leq \int_{0}^{1 / 2} \psi^{-1}\left(\frac{1}{\alpha-1} s^{-(\alpha-1)}\right) d s \\
& =\int_{0}^{1 / 2}\left(\frac{s^{-(\alpha-1)}}{\alpha-1}\right)^{1 /(q-1)} d s \\
& =\left.\frac{q-1}{(\alpha-1)^{1 /(q-1)}(q-\alpha)} s^{(q-\alpha) /(q-1)}\right|_{0} ^{1 / 2}<\infty,
\end{aligned}
$$

since $q-\alpha>0$ and $q-1>0$. The continuity of $h(t)=t^{-\alpha}$ on $[1 / 2,1]$ obviously implies that $\int_{1 / 2}^{1} \psi^{-1}\left(\int_{1 / 2}^{s} \tau^{-\alpha} d \tau\right) d s<\infty$.
Similarly, we can show that $h(t)=t^{-\beta} \in \mathscr{H}_{\psi}$. We now check the conditions on the nonlinear terms. Both $f^{1}(u, v)=(u+$ $v)^{p-q}+1$ and $f^{2}(u, v)=u^{q-1}\left(1-e^{-v}\right)$ satisfy $(\mathrm{F})$ and

$$
\begin{aligned}
f_{0}^{1} & =\lim _{\|(u, v)\| \rightarrow 0} \frac{f^{1}(u, v)}{\varphi(\|(u, v)\|)} \\
& =\lim _{\|(u, v)\| \rightarrow 0} \frac{(u+v)^{p-q}+1}{(u+v)^{q-1}\left[(u+v)^{p-q}+1\right]}=\infty, \\
f_{\infty}^{1} & =\lim _{\|(u, v)\| \rightarrow \infty} \frac{f^{1}(u, v)}{\varphi(\|(u, v)\|)} \\
& =\lim _{\|(u, v)\| \rightarrow \infty} \frac{1}{(u+v)^{q-1}}=0, \\
f_{0}^{2} & =\lim _{\|(u, v)\| \rightarrow 0}\left(1-e^{-v}\right) \cdot \frac{(u+v)^{p-1}+(u+v)^{q-1}}{\left(1-e^{q-1}\right)=0,} \\
& \leq \lim _{\|(u, v)\| \rightarrow 0}\left(1-e^{-1}\right.
\end{aligned}
$$

$$
\begin{aligned}
f_{\infty}^{2} & =\lim _{\|(u, v)\| \rightarrow \infty}\left(1-e^{-v}\right) \cdot \frac{u^{q-1}}{(u+v)^{p-1}+(u+v)^{q-1}} \\
& \leq \lim _{\|(u, v)\| \rightarrow \infty}\left(1-e^{-v}\right) \cdot \frac{(u+v)^{q-1}}{(u+v)^{p^{-1}}+(u+v)^{q-1}} \\
& \leq \lim _{\|(u, v)\| \rightarrow \infty} \frac{1}{(u+v)^{p-q}+1}=0 .
\end{aligned}
$$

Thus, $\mathbf{f}_{0}=f_{0}^{1}+f_{0}^{2}=\infty, \mathbf{f}_{\infty}=f_{\infty}^{1}+f_{\infty}^{2}=0$. Consequently, by Theorem 2, we see that problem $\left(E_{1}\right)$ has at least one positive solution.

Example 14. Consider the following $\varphi$-Laplacian system:

$$
\begin{gathered}
\varphi\left(u^{\prime}\right)^{\prime}+t^{-5 / 4}(u+v)^{1 / 2}=0, \\
\varphi\left(v^{\prime}\right)^{\prime}+t^{-6 / 5}\left(1-e^{-(u+v)}\right)(u+v)^{1 / 3}=0, \quad t \in(0,1), \\
u(0)=v(0)=u(1)=v(1)=0,
\end{gathered}
$$

where $\varphi(x)=x^{1 / 3}, x \in \mathbb{R}$, is an odd increasing homeomorphism. By the homogeneity of $\varphi$, taking $\psi(\sigma)=\gamma(\sigma) \equiv \varphi(\sigma)$, we see that condition $(\mathrm{A})$ is satisfied. Consider

$$
\begin{aligned}
\int_{0}^{1 / 2} & \psi^{-1}\left(\int_{s}^{1 / 2} \tau^{-5 / 4} d \tau\right) d s \\
& =\int_{0}^{1 / 2} \psi^{-1}\left(4\left(s^{-1 / 4}-2^{1 / 4}\right)\right) d s \\
& =\int_{0}^{1 / 2}\left(4\left(s^{-1 / 4}-2^{1 / 4}\right)\right)^{3} d s \\
& \leq 64 \int_{0}^{1 / 2} s^{-3 / 4} d s=\left.256 s^{1 / 4}\right|_{0} ^{1 / 2}<\infty
\end{aligned}
$$


and the continuity of $h(t)=t^{-5 / 4}$ on $[1 / 2,1]$ implies that $h(t)=t^{-5 / 4} \in \mathscr{H}_{\psi}$. Similarly, we can show that $h(t)=t^{-6 / 5} \in$ $\mathscr{H}_{\psi}$. For the nonlinear terms, both $f^{1}(u, v)=(u+v)^{1 / 2}$ and $f^{2}(u, v)=\left(1-e^{-(u+v)}\right)(u+v)^{1 / 3}$ satisfy condition $(\mathrm{F})$ and

$$
\begin{aligned}
f_{0}^{1} & =\lim _{\|(u, v)\| \rightarrow 0} \frac{f^{1}(u, v)}{\varphi(\|(u, v)\|)} \\
& =\lim _{\|(u, v)\| \rightarrow 0} \frac{(u+v)^{1 / 2}}{(u+v)^{1 / 3}} \\
& =\lim _{\|(u, v)\| \rightarrow 0}(u+v)^{1 / 6}=0, \\
f_{\infty}^{1} & =\lim _{\|(u, v)\| \rightarrow \infty} \frac{f^{1}(u, v)}{\varphi(\|(u, v)\|)} \\
& =\lim _{\|(u, v)\| \rightarrow \infty}(u+v)^{1 / 6}=\infty, \\
f_{0}^{2} & =\lim _{\|(u, v)\| \rightarrow 0}\left(1-e^{-(u+v)}\right) \cdot \frac{(u+v)^{1 / 3}}{(u+v)^{1 / 3}} \\
& =\lim _{\|(u, v)\| \rightarrow 0}\left(1-e^{-(u+v)}\right)=0, \\
f_{\infty}^{2} & =\lim _{\|(u, v)\| \rightarrow \infty}\left(1-e^{-(u+v)}\right)=1 .
\end{aligned}
$$

Thus, $\mathbf{f}_{0}=f_{0}^{1}+f_{0}^{2}=0, \mathbf{f}_{\infty}=f_{\infty}^{1}+f_{\infty}^{2}=\infty$. Consequently, by Theorem 2, we see that problem $\left(E_{2}\right)$ has at least one positive solution.

\section{Conflict of Interests}

The authors declare that there is no conflict of interests for this paper.

\section{Authors' Contribution}

All authors have equally contributed in obtaining new results in this paper and also read and approved the final paper.

\section{Acknowledgment}

This research was supported by Basic Science Research Program through the National Research Foundation of Korea (NRF) funded by the Ministry of Education, Science, and Technology (no. 2012005767).

\section{References}

[1] J. I. Díaz, Nonlinear Partial Differential Equations and Free Boundaries. Vol. I, vol. 106 of Research Notes in Mathematics, Pitman, Boston, Mass, USA, 1985, Elliptic equations.

[2] P. Drábek, A. Kufner, and F. Nicolosi, Quasilinear Elliptic Equations with Degenerations and Singularities, vol. 5 of de Gruyter Series in Nonlinear Analysis and Applications, Walter de Gruyter \& Co., Berlin, Germany, 1997.

[3] R. Glowinski and J. Rappaz, "Approximation of a nonlinear elliptic problem arising in a non-Newtonian fluid flow model in glaciology," Mathematical Modelling and Numerical Analysis, vol. 37, no. 1, pp. 175-186, 2003.

[4] D. O'Regan, "Some general existence principles and results for $\left(\phi\left(y^{\prime}\right)\right)^{\prime}=q f\left(t, y, y^{\prime}\right), 0<t<1$," SIAM Journal on Mathematical Analysis, vol. 24, no. 3, pp. 648-668, 1993.

[5] D. Bai and Y. Chen, "Three positive solutions for a generalized Laplacian boundary value problem with a parameter," Applied Mathematics and Computation, vol. 219, no. 9, pp. 4782-4788, 2013.

[6] X. Cheng and H. Lü, "Multiplicity of positive solutions for a $\left(p_{1}, p_{2}\right)$-Laplacian system and its applications," Nonlinear Analysis: Real World Applications, vol. 13, no. 5, pp. 2375-2390, 2012.

[7] J. M. do Ó, S. Lorca, J. Sánchez, and P. Ubilla, "Positive radial solutions for some quasilinear elliptic systems in exterior domains," Communications on Pure and Applied Analysis, vol. 5, no. 3, pp. 571-581, 2006.

[8] J. Henderson and H. Wang, "Nonlinear eigenvalue problems for quasilinear systems," Computers \& Mathematics with Applications, vol. 49, no. 11-12, pp. 1941-1949, 2005.

[9] E. K. Lee and Y. H. Lee, "A multiplicity result for generalized Laplacian systems with multiparameters," Nonlinear Analysis: Theory, Methods \& Applications A: Theory and Methods, vol. 71, no. 12, pp. 366-376, 2009.

[10] H. Wang, "On the number of positive solutions of nonlinear systems," Journal of Mathematical Analysis and Applications, vol. 281, no. 1, pp. 287-306, 2003.

[11] H. Wang, "On the structure of positive radial solutions for quasilinear equations in annular domains," Advances in Differential Equations, vol. 8, no. 1, pp. 111-128, 2003.

[12] R. P. Agarwal, D. O’Regan, and S. Staněk, "General existence principles for nonlocal boundary value problems with $\varphi$ Laplacian and their applications," Abstract and Applied Analysis, vol. 2006, Article ID 96826, 30 pages, 2006.

[13] R. P. Agarwal, D. O’Regan, and S. Staněk, "Dead cores of singular Dirichlet boundary value problems with $\varphi$-Laplacian," Applications of Mathematics, vol. 53, no. 4, pp. 381-399, 2008.

[14] R. P. Agarwal, D. O’Regan, and S. Staněk, "Positive and dead core solutions of singular Dirichlet boundary value problems with $\varphi$-Laplacian," Computers \& Mathematics with Applications, vol. 54, no. 2, pp. 255-266, 2007.

[15] I. Sim and Y. H. Lee, "A new solution operator of onedimensional $p$-Laplacian with a sign-changing weight and its application," Abstract and Applied Analysis, vol. 2012, Article ID 243740, 15 pages, 2012.

[16] K. Deimling, Nonlinear Functional Analysis, Springer, New York, NY, USA, 1985.

[17] M. A. Krasnoselskii, Positive Solutions of Operator Equations, Noordhoff, Groningen, The Netherlands, 1964. 


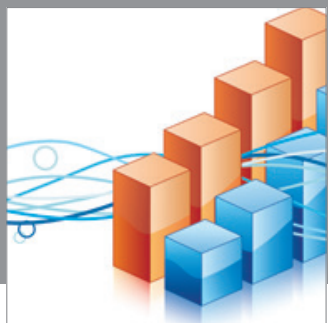

Advances in

Operations Research

mansans

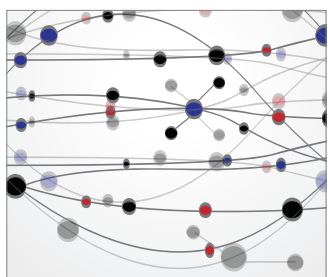

The Scientific World Journal
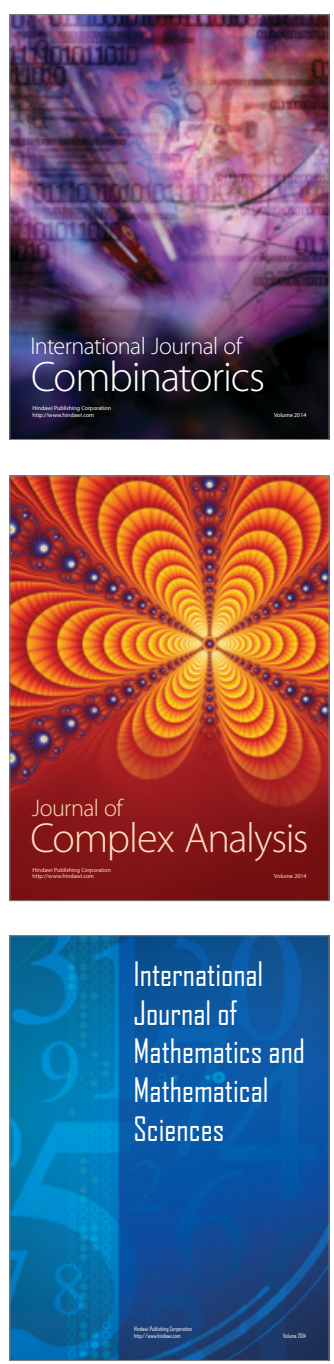
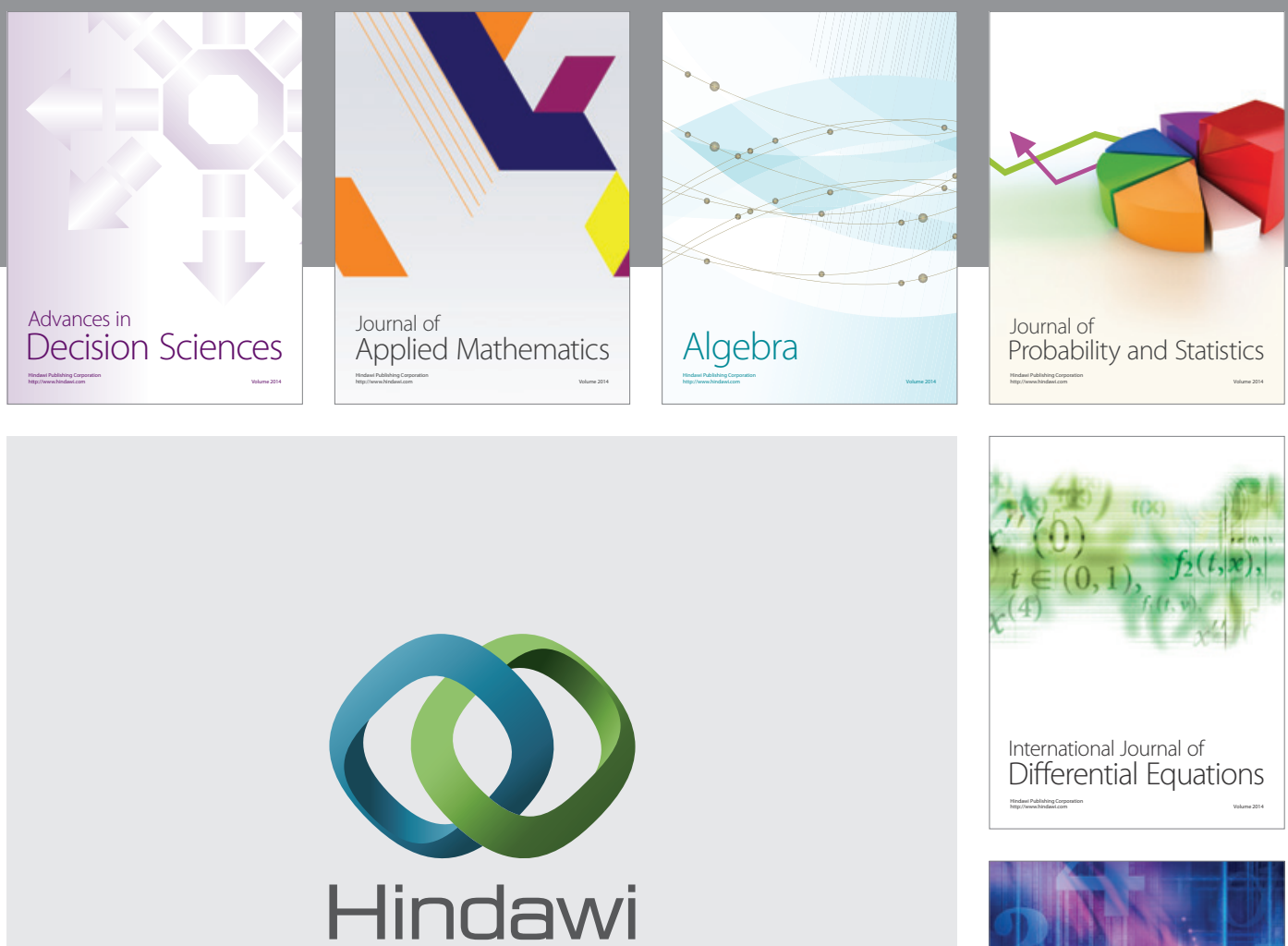

Submit your manuscripts at http://www.hindawi.com
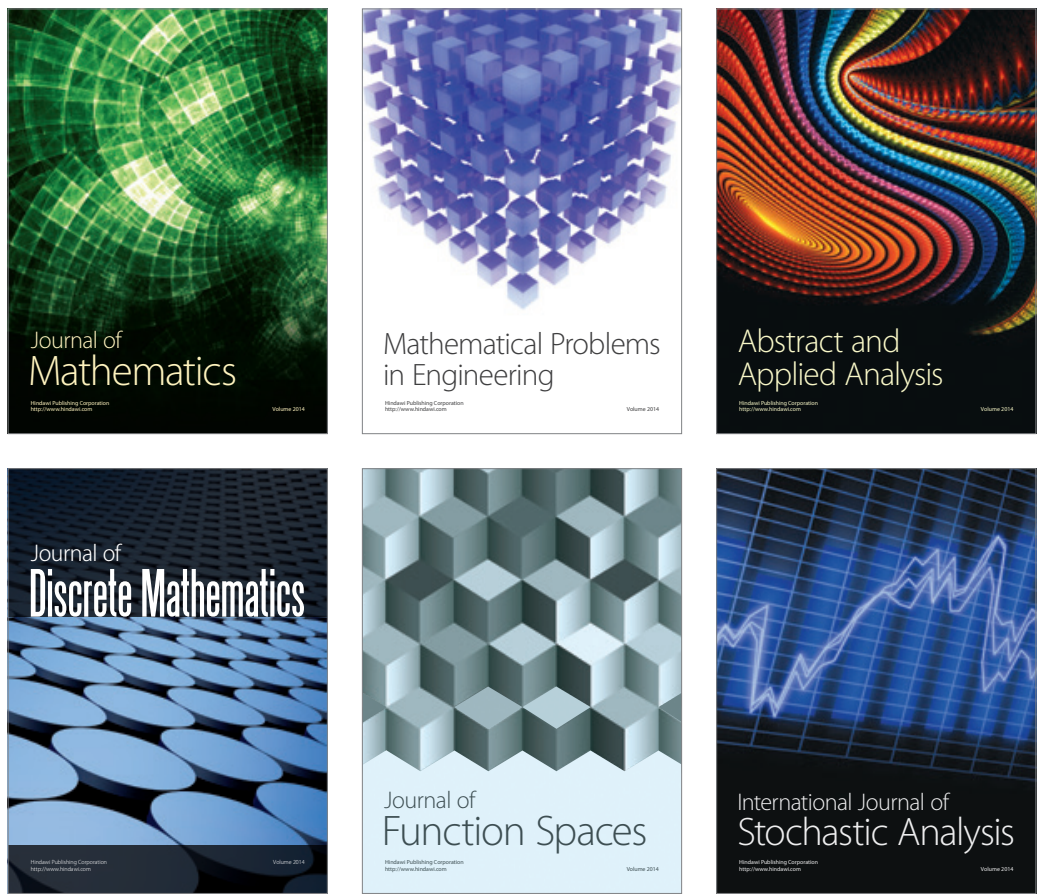

Journal of

Function Spaces

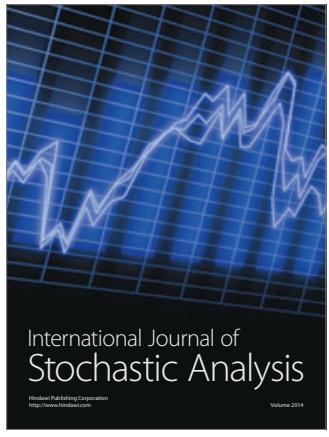

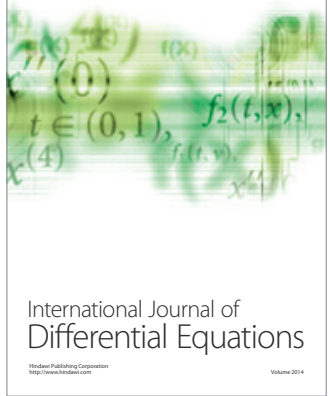
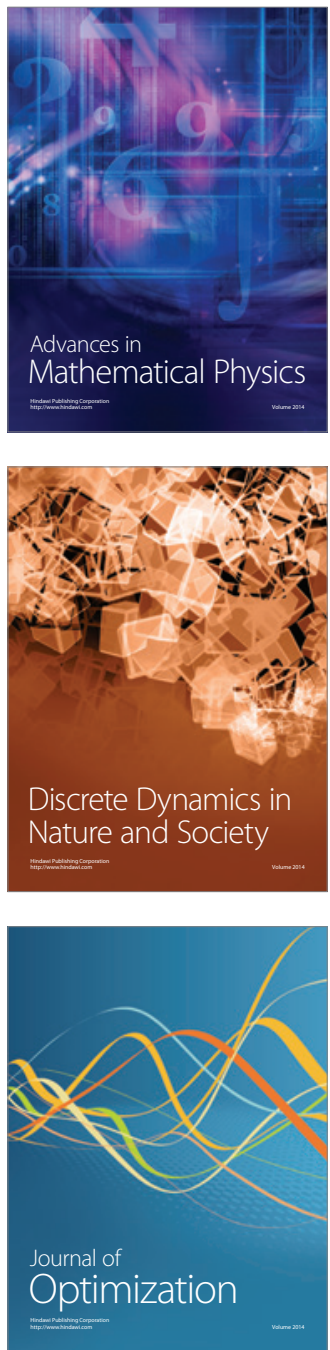